\title{
162. Mechanism of Action Potential Production in the Nerve Cell of a Puffer
}

\author{
By Susumu Hagrwara and Nozomu SaIto \\ Department of Physiology, Tokyo Medical and Dental University, Tokyo \\ (Comm. by T. Furuhata, M.J.A., Dec. 12, 1957)
}

A group of giant nerve cells are found on the dorsal surface of the medulla of a puffer. According to their large sizes (100 to $250 \mu$ in diameter) and to their superficial location in the medulla they are uniquely fit to the investigation with the multi-internal-electrode technique.*) The present work is planned to elucidate the mechanism of action potential production in the nerve cell by using the double-electrode technique, voltage-clamp technique, and others.

Although those giant cells were found in several species of puffers obtainable along the Pacific coast of Japan, Spheroides vermicularis was mainly used because of good visibility of individual cells. A fish was narcotised with tubocurarine chloride $(30-40 \mathrm{mg}$ per $\mathrm{kg}$ ) and the artificial respiration was performed by circulating sea water from its mouth. After exposing the dorsal surface of the medulla two internal microelectrodes were inserted into the same cell under the visual control through a binocular microscope. In the first type of experiment the change of the membrane potential was observed with one electrode while a constant current pulse was applied through the membrane by means of another electrode. In the second type of experiment a negative feed-back system was inserted between the recording and the current electrodes so as that the membrane potential was kept at a desired level (voltage clamp). The feed-back system used was similar to that used in the previous work $^{1)}$ but a few modifications were made for the unavoidable high resistance of internal current electrode.

1. Constant current stimulation. The resting potential of the cell ranges between 55 and $65 \mathrm{mV}$. A constant current pulse passed through the cell membrane in the outward direction causes a depolarization of the membrane. When the depolarization reaches a certain value $(18-25 \mathrm{mV})$ it gives origin to a spike potential (Fig. 1, A). The spike potential has a peak voltage of $80-100 \mathrm{mV}$ and is associated with a positive after-potential or an undershoot. At the peak of the undershoot the potential at the inside of the cell becomes approximately $18 \mathrm{mV}$ more negative than at rest. The value of depolarization

*) Recently the work on the similar nerve cell of an Atlantic puffer was reported by S. M. Crain, M. V. L. Bennett, and H. Grundfest (Biol. Bull., 113, 342 (1957)). 

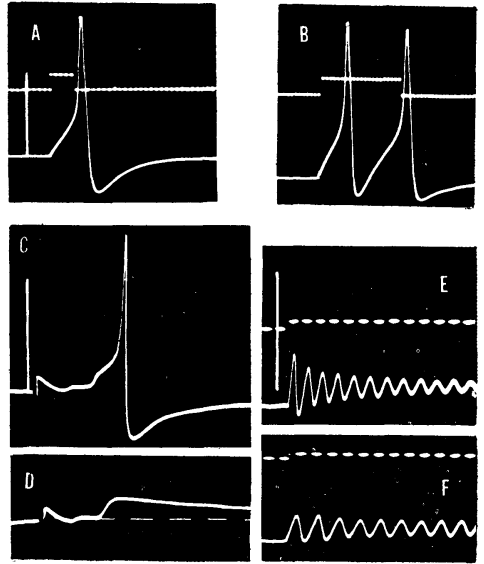

Fig. 1

A: Action potential produced by a brief outward constant current pulse. The intensity of the current is just above the threshold. B: Stimulation with a constant current pulse of a long duration. The second spike appears. The intensity of the current is the same to that in A. Blanking in current recording $1 \mathrm{msec}$ interval in $\mathrm{A}$ and also in B. C: Action potential produced by a presynaptic stimulation. D: Synaptic potential unassociated with a spike. Time marker: 10 msec interval in $\mathrm{C}$ and D. E and F: Oscillatory potential changes produced by outward current pulses. Time marker: $10 \mathrm{msec}$ interval. Potential calibration in all records: $50 \mathrm{mV}$ critical for the spike is independent of the time to reach it. When the current is continued after the undershoot of the first spike the redevelopment of depolarization appears and the second spike rises from the depolarization when it reaches the value approximately similar to the critical depolarization of the first spike (Fig. 1, B). Thus repetitive spikes occur and the interval between the successive two spikes decreases with the intensity of the applied outward current.

When the cell is under poor conditions such as under oxygen lack, it does not show an all-or-none spike. The response produced by a brief outward pulse is a type of local potential. For a pulse of a long duration the potential change of the membrane is a sinusoidal oscillation (Fig. 1, E and F). The amplitude, the frequency, and the rate of damping of the oscillation are determined by the intensity of the applied current.

With a constant current pulse of an inward direction the potential change is an increase of the membrane potential and the time course of the potential change represents a charging curve of a simple leaky condenser. This fact shows that the internal protoplasm of the cell is equipotential all over and furthermore it also shows that the applied current mainly flows through the membrane of the nerve cell even though the cell is connected with an axon. The time constant and the resting membrane resistance obtained from the above time course are 4-6 msec and $0.6-2.5 \mathrm{M} \Omega$ respectively. Measuring the diameter of the cell we obtained about $5-15 \mu \mathrm{F}$ and $0.5-1 \mathrm{~K} \Omega$ for the specific values of the membrane capacity and the membrane resistance.

2. Constant potential stimulation (voltage clamp). Since the whole membrane of the cell is under the space clamp and since the applied current is mainly flows through the membrane of the cell, the current-potential relation of the cell membrane can be obtained with the voltage-clamp technique. 

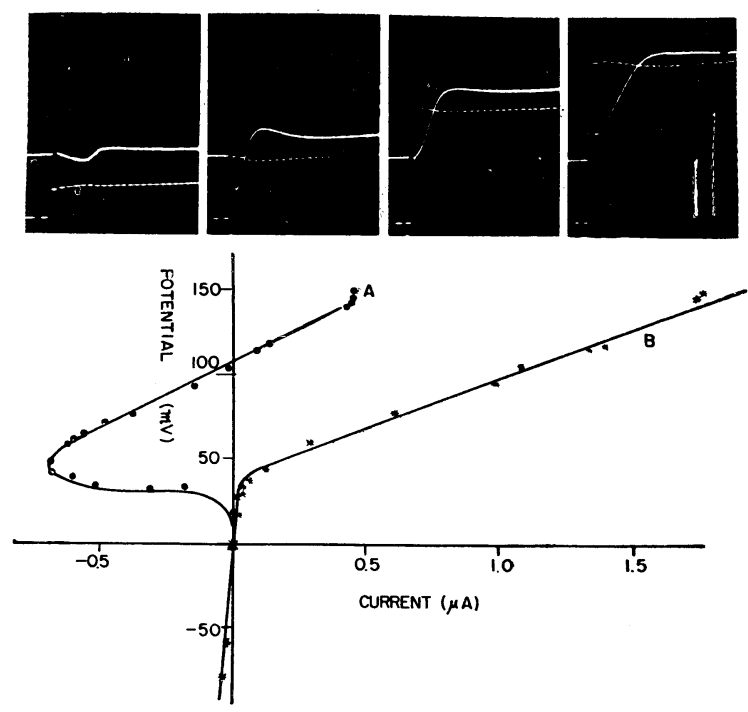

Fig. 2

Upper: Records of membrane current associated with a rectangular depolarization of the cell membrane. The lower dotted trace shows the membrane potential and the upper trace shows the current. Potential calibration: $100 \mathrm{mV}$. Current calibration: $1 \mu \mathrm{A}$. Blanking: $1 \mathrm{msec}$ interval. Lower: Relation between the membrane potential and the membrane current. Depolarization: Positive. Outward current: Positive. A: Current intensity at the peak of initial surge. B: Intensity of the final steady current
When the clamped membrane potential is a hyperpolarized level, the current is an inward constant current preceded by a capacitative surge. The relation between the current and the potential is linear as shown in Fig. 2 (lower). When the membrane potential is clamped at a depolarized level which is below the critical depolarization for the spike (below $20 \mathrm{mV}$ ) the current is a constant outward current. The ratio between the current and the potential is the same to that obtained with the hyperpolarization as shown in the figure. These results show that

in the membrane of the present cell no obvious delayed rectification exists at the subthreshold depolarization. When the depolarization is just above $20 \mathrm{mV}$, an initial small inward surge follows the capacitative surge and the former is followed by an outward current, but the outward current is still similar to that expected from the resting membrane resistance. Further increase of depolarization increases the initial inward surge and at a certain membrane potential the amplitude of the initial inward surge reaches a maximum. Approximately at this potential level a gradual increase of the outward current preceded by the initial surge begins to be observed. While the membrane potential is kept constant, the outward current increases and finally reaches a value which is much larger than that expected from the resting membrane resistance. In other words the delayed rectification appears from this level of depolarization. For the depolarization higher than this level the amplitude of the initial surge as well as the final steady current shows a linear relation with the membrane potential. When the clamped membrane potential is about $100 \mathrm{mV}$ above the resting level the initial surge changes its 
sign from inward to outward and this level is somewhat higher than the potential level at the peak of the spike, but the difference is very small. Although the above current-potential relation is, in general, similar to that obtained with a squid giant axon by the previous workers, ${ }^{122}$ there are a few differences between them. One of the differences is that the level of depolarization at which the delayed rectification appears is somewhat higher than the level at which the initial inward surge appears. The membrane resistance at the peak of the spike can be estimated from the slope of the current-potential relation. It ranges between 30 and $90 \mathrm{~K} \Omega$ and is $1 / 20$ to $1 / 30$ of the resting membrane resistance.

3. Stimulation through synapse. When the spinal cord of the fish is stimulated with a short current pulse a spike potential appears in the nerve cell (Fig. 1, C). Such a spike is invariably preceded by a small pre-potential which represents the initial part of a synaptic potential. By changing certain conditions it is possible to make the amplitude of the synaptic potential small enough not to produce a spike. These abortive synaptic potentials show a very slow time course (the half decay time is $20-30 \mathrm{msec}$ ). Sometimes a series of similar synaptic potentials associated with and without a spike is observed in an unstimulated preparation. Therefore, the present cell seems to receive messages from the afferent fibers in the spinal cord under normal condition.

The authors wish to express their indebtedness to Prof. Y. Katsuki for his criticisms, to Mr. K. Ogura for his technical assistances in the electrical engineering, to Dr. E. Berkwitz for informing the presence of those giant cells in the puffer brain.

\section{References}

1) I. Tasaki and S. Hagiwara: J. Gen. Physiol., 40, 859 (1957).

2) A. L. Hodgkin and A. F. Huxley: J. Physiol., 116, 449 (1952). 